\section{Prevalence of Anemia in Preschool Aged Children Living in Dominica}

\section{Abstract}

Introduction: Anemia affects more than one billion people worldwide, with pregnant women and children under five years of age comprising the vast majority of those afflicted. The development of anemia is multifactorial and could stem from a variety of factors. Nutritional deficiencies especially iron, but also folate, vitamin B12, vitamin A, and protein appear to be the biggest factor. Diagnosing anemia in children under five years of age is of utmost importance because these nutrients are essential for their rapid phase of growth and development.

Objective: The purpose of this study was to determine the prevalence of anemia in children under six years of age living in Dominica.

Methods: This was a retrospective, descriptive study, examining the prevalence of anemia in preschool children, aged two to six years, living in Dominica from January 1, 2012 to December 31, 2015. All available medical records of the children were collected and analyzed using Stata version 14 . There were no exclusion criteria.

Results and discussion: A sample population of 635 children was included in our study. The majority of children was females $(54.7 \%)$, at least 3.5 years old $(60.6 \%)$, and attended preschool in the parish of Saint George (58.7\%). Our study determined that the prevalence of anemia was $19.3 \%$. Additionally, we found that $22 \%$ of anemic children had a mean corpuscular volume below $75 \mathrm{fL}$, and $78 \%$ of anemic children had a mean corpuscular volume between $76 \mathrm{fL}$ and $99 \mathrm{fL}$.

Conclusion: The data in this study demonstrated that childhood anemia in Dominica is widely prevalent, and represents a public health issue that needs to be addressed. According to our data, $22 \%$ of anemic children had a mean corpuscular volume below $75 \mathrm{fL}$ indicating a microcytic anemia. Microcytic anemia could suggest diseases such as iron deficiency and thalassemia among many other causes. Additionally, $78 \%$ of anemic children had a mean corpuscular volume between $76 \mathrm{fL}$ and $99 \mathrm{fL}$ indicating a normocytic anemia. Normocytic anemia could suggest chronic disease and sickle cell anemia among many others. Due to limited resources, we were unable to determine the specific cause of anemia; however, future studies should be conducted so that strategies could be developed, on a national level, to resolve this childhood burden.

Keywords: Normocytic anemia; Intestinal helminths; Hemoglobinopathies; Thalassemia

\section{Brittany Noel Robles*, Adelbert B James, Raffaele Macri, Mai Xiong, Sabeen Jafri, Alyssa Vitale, Victoria Beata, Sarah Faheem and Rhonda McIntyre-Francis}

Mount Sinai Health System, Icahn School of Medicine at Mount Sinai, New York, USA

Corresponding author:

Brittany Noel Robles

” brittanynoelrobles@gmail.com

Mount Sinai Health System, Icahn School of Medicine at Mount Sinai, 417 East 70th St New York, USA.

Tel: +1 212-241-6696

Citation: Robles $B N$, James $A B$, Macri R, Xiong M, Jafri S, et al. (2017) Prevalence of Anemia in Preschool Aged Children Living in Dominica. J Pediatr Care. Vol. 3 No. 3:12

Received: July 25, 2017; Accepted: August 11, 2017; Published: August 15, 2017

\section{Introduction}

Anemia affects more than one billion people worldwide, with pregnant women and children under five years of age comprising the vast majority of those afflicted. The development of anemia is multifactorial and could stem from a variety of factors. Nutritional deficiencies especially iron, but also folate, vitamin B12, vitamin A, and protein appear to be the biggest factor [1-4]. Infectious diseases, such as malaria and intestinal helminths, as well as environmental pollutants, such as lead, could also lead 
to the development of anemia [1-4]. Furthermore, anemia could arise due to abnormalities in the genetic makeup of an individual leading to one of many types of hemoglobinopathies [1-5]. Although there are many factors that influence the development of anemia, studies have shown that nutritional deficiencies, specifically due to a decrease in the amount of iron rich food consumed, appears to be the most common cause of anemia, with over one million cases diagnosed [2-4].

Anemia during the first one thousand days of a child's life is of special importance because it is during this time period that neonates are extremely susceptible to illness. Beginning at birth, infants undergo a rapid phase of growth and development which relies on many vitamins and nutrients. Of those vitamins and nutrients, iron is particularly important for the adequate development of the brain and neuronal system [2]. When there is a deficiency of iron, anemia occurs which could cause a plethora of problems such as impairments in school, intellectual development, physical growth, and ability to fight infection [3]. Often times, without an early intervention these problems could have a lifelong impact.

The World Health Organization (WHO) has developed a classification system for determining the severity of anemia based on the population prevalence. The problem is considered severe if the anemia prevalence rate is $>40 \%$, moderate if the anemia prevalence rate is $20 \%$ to $39.9 \%$, and mild if the prevalence rate is $5 \%$ to $19.9 \%$ [6]. In 2011, Dominica had an anemia prevalence rate of $37 \%$ in children under five years of age 8 . Although Dominica had a prevalence rate lower than the global prevalence rate of $47 \%$ the country still had a moderate level severity. Without an intervention, the prevalence could continue to grow, further burdening the society as a whole.

Due to the limited data on childhood anemia in Dominica, the purpose of this study was to perform a descriptive study to determine the prevalence of anemia among preschool aged children.

\section{Methods}

Our study was a non-concurrent medical record review, examining the prevalence of anemia in preschool children, aged two to six years, living in Dominica from January 1, 2012 to December 31, 2015.

\section{Data collection}

Before attending pre-school, children in Dominica are required to have basic blood work done to ensure they are healthy enough to enroll. The blood work is drawn at a laboratory, and the results are placed in the child's medical record. The medical records are then stored at the child's pre-school.

In October 2016, the chief nurse in Dominica visited each district and collected hard copies of the children's medical records. The chief nurse then stored the medical records at the main hospital, located in the city of Roseau. At the hospital, medical students from Ross University School of Medicine reviewed and recorded the data.
The medical records were reviewed from November 1, 2016 to December 1, 2016. A standard form and password protected excel data-base was created and used to collect and manage the data. The following data was collected from the medical records: Date of birth, gender, pre-school attended, parish the pre-school was located in, height in centimeters, weight in kilograms, hemoglobin measured in deci-liters, hematocrit measured in percent, mean corpuscular volume measured in fluid liters, red blood cell distribution width measure in percent, and the presence or absence of stool ova and parasites.

\section{Sample}

The medical students reviewed all 696 medical records that were stored at the main hospital in the city of Roseau. Sixtyone children were eliminated due to incomplete data. The final sample size was 635 children. There were no exclusion criteria.

\section{Study variables}

The dependent variable was hemoglobin. The independent variables were age, gender, parish in which the pre-school was located, height, weight, hematocrit, mean corpuscular volume, red blood distribution width, and stool ova and parasites. The complete data collection sheet includes DOB, Pre-school attended gender (male/female), height $(\mathrm{cm})$, weight $(\mathrm{kg})$, hemoglobin $(\mathrm{g} /$ $\mathrm{dL}$ ), hematocrit $(\%)$, mean corpuscular volume $(\mathrm{fL})$, red blood cell distribution width (\%), stool ova and parasites.

\section{Statistical analysis}

The dataset was imported into Stata statistical program-version 14 to perform data analyses. We categorized each variable and computed frequency tables and two-way tables with measures of associations. Pearson's chi-squared, odds ratio, 95\% confidence intervals and $p$ values were computed to determine statistical significance between variables. A p-value of less than 0.05 was considered statistically significant.

\section{Ethical issues}

The Institutional Review Board at The Icahn School of Medicine at Mount Sinai and the Ethics Committee of Dominica approved the investigation, with a waiver of consent. This study was conducted in accordance with the Belmont Report and the tenets put forth in the Declaration of Helsinki.

\section{Results}

Table 1 describes the demographic and laboratory values of the study sample. A total of 635 children were included in our study. The majority of children was females (54.7\%), at least 3.5 years old $(60.6 \%)$, and attended pre-school in the parish of Saint George (58.7\%).

Figure 1 illustrates the percentage of children included in the study who attended pre-school in the respective parish. The preschools were located in all ten parishes of Dominica. The majority of children, $349(58.7 \%)$ attended pre-school in the parish of Saint George and the minority of children, $6(1.0 \%)$ attended preschool in the parish of Saint Peter. 
Table 1 Characteristics of study participants.

\begin{tabular}{|c|c|c|}
\hline Variables & N (635) & (\%) \\
\hline \multicolumn{3}{|c|}{ Sex* } \\
\hline Male & 283 & -45.2 \\
\hline \multirow[t]{2}{*}{ Female } & 343 & -54.7 \\
\hline & Age & \\
\hline$<3.5$ years & 250 & -39.3 \\
\hline$\geq 3.5$ years & 385 & -60.6 \\
\hline \multicolumn{3}{|c|}{ Parish* } \\
\hline Saint Andrew & 8 & -1.3 \\
\hline Saint George & 349 & -58.7 \\
\hline Saint John & 52 & -8.7 \\
\hline Saint Joseph & 62 & -10.4 \\
\hline Saint Luke & 7 & -1.1 \\
\hline Saint Mark & 10 & -1.6 \\
\hline Saint Patrick & 14 & -2.3 \\
\hline Saint Paul & 70 & -11.7 \\
\hline Saint David & 16 & -2.6 \\
\hline Saint Peter & 6 & -1 \\
\hline \multicolumn{3}{|c|}{ Hemoglobin*o } \\
\hline$<11.5 \mathrm{~g} / \mathrm{dL}$ & 115 & -19.3 \\
\hline$\geq 11.5 \mathrm{~g} / \mathrm{dL}$ & 480 & -80.6 \\
\hline \multicolumn{3}{|c|}{ Hematocrit* } \\
\hline$<35 \%$ & 100 & -37.4 \\
\hline$\geq 35 \%$ & 167 & -62.5 \\
\hline \multicolumn{3}{|c|}{ Mean corpuscular volume* } \\
\hline$<75 \mathrm{fL}$ & 57 & -22.4 \\
\hline$\geq 76$ to $99 \mathrm{fL}$ & 197 & -77.4 \\
\hline \multicolumn{3}{|c|}{ Red blood cell distribution width* } \\
\hline$<15 \%$ & 238 & -94.4 \\
\hline$\geq 15 \%$ & 14 & -5.5 \\
\hline \multicolumn{3}{|c|}{ Stool ova and parasite* } \\
\hline Negative & 586 & -95.6 \\
\hline Positive & 27 & -4.4 \\
\hline \multicolumn{3}{|c|}{$\begin{array}{l}\text { Note }-{ }^{*} \text { missing data; }{ }^{\ominus} \text { the definition of anemia varies based on various } \\
\text { sources; however, after consulting with Dr. Edwin Forman, a Pediatric } \\
\text { Hematologist-Oncologist at Mount Sinai Medical Center in New York } \\
\text { City, he deemed } 11.5 \mathrm{~g} / \mathrm{dL} \text { as an appropriate measurement of anemia. }\end{array}$} \\
\hline
\end{tabular}

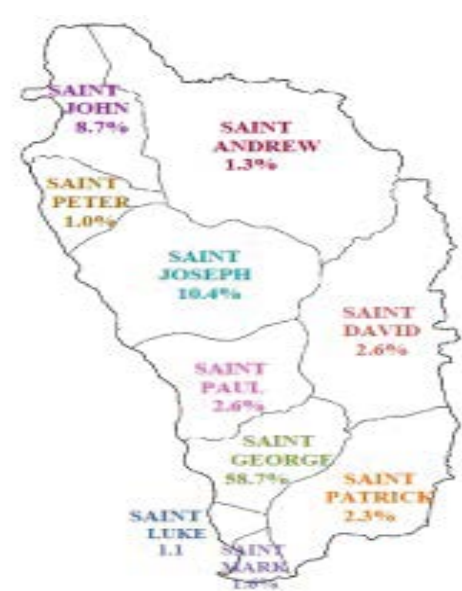

Figure 1 Percentage of children aged 2 years to 6 years old included in the study that attended pre-school in the respective parish.
The presence of anemia, measured by a hemoglobin level of less than $11.5 \mathrm{~g} / \mathrm{dL}$, was found in 115 (19.3\%) children compared to $480(80.6 \%)$ children who had hemoglobin levels greater than or equal to $11.5 \mathrm{~g} / \mathrm{dL}$. Of the 267 children who had their hematocrit level recorded, 100 (37.4\%) children had hematocrit values below $35 \%$ compared to 167 (62.5\%) children who had hematocrit values greater than or equal to $35 \%$. Additionally, there were 254 children who had their Mean Corpuscular Volume (MCV) recorded. Of those 254 children, 57 (22.4\%) had MCV levels below $75 \mathrm{fL}$, compared to 197 children (77.5\%) who had MCV levels between $76 \mathrm{fL}$ to $99 \mathrm{fL}$. There were 0 children who had a MCV level greater than or equal to $100 \mathrm{fL}$ (Tables 1 and 2) (Figure 1).

Table 2 depicts the differences between children who had a hemoglobin less than $11.5 \mathrm{~g} / \mathrm{dL}$ and those who had a hemoglobin greater than or equal to $11.5 \mathrm{~g} / \mathrm{dL}$. The majority of children, 474 , had a hemoglobin level greater than or equal to $11.5 \mathrm{~g} / \mathrm{dL}$. Of those children who had a hemoglobin value greater than or equal to $11.5 \mathrm{~g} / \mathrm{dL}$, the majority was greater than or equal to 3.5 years of age. It was concluded that children who had a hematocrit level of $<35 \%$ were 48 times more likely to have a hemoglobin value of $<11.5 \mathrm{~g} / \mathrm{dL}$ than children with a hematocrit value of $\geq 35 \%, \mathrm{p}$ value $<0.000$ (OR=48, $95 \% \mathrm{Cl}(16.4,1.92 .2)$. Additionally, it was determined that children who had a mean corpuscular volume of $<75 \mathrm{fL}$ were 2.6 times more likely to have a hemoglobin value of $<11.5 \mathrm{~g} / \mathrm{dL}$ than children who had a mean corpuscular volume $\geq 76 \mathrm{fL}$ to $99 \mathrm{fL}, \mathrm{p}$ value 0.003 (OR=2.6, $95 \% \mathrm{Cl}(1.28,5.21)$. Furthermore, our study concluded that children who had a red blood cell distribution width less than 15 , were 0.3 times less likely to have a hemoglobin level less than $11.5 \mathrm{~g} / \mathrm{dL}(\mathrm{OR}=0.259$; $95 \% \mathrm{Cl}(0.074,0.916)$.

Figure 2 displays the percentage of children included in our study who attended pre-school in a parish with a hemoglobin level below $11.5 \mathrm{~g} / \mathrm{dL}$, indicating anemia (Figure 2).

Table 2 Hemoglobin levels in study participants.

\begin{tabular}{|c|c|c|c|c|}
\hline Variables & $\begin{array}{c}\mathrm{Hg}<11.5 \mathrm{~g} / \\
\quad \mathrm{dL}(\mathrm{n})\end{array}$ & $\begin{array}{c}\mathrm{Hg} \geq 11.5 \mathrm{~g} / \\
\mathrm{dL}(\mathrm{n})\end{array}$ & Total (n) & $p$ value \\
\hline Female & 62 & 258 & 320 & \multirow{3}{*}{$p=0.859$} \\
\hline Male & 50 & 216 & 266 & \\
\hline Age $<3.5$ yo & 46 & 185 & 231 & \\
\hline $\begin{array}{c}\text { Age } \geq 3.5 \\
\text { years }\end{array}$ & 69 & 295 & 364 & $p=0.773$ \\
\hline $\mathrm{MCV}<75$ & 21 & 36 & 57 & \multirow[t]{2}{*}{$p=0.003^{\prime}$} \\
\hline$M C V \geq 76-99$ & 36 & 161 & 197 & \\
\hline Hct $<35 \%$ & 55 & 45 & 100 & \multirow{2}{*}{$p=0.000^{*}$} \\
\hline Hct $\geq 35 \%$ & 4 & 160 & 164 & \\
\hline RDW $<15 \%$ & 49 & 189 & 238 & \multirow{2}{*}{$p=0.010^{*}$} \\
\hline $\mathrm{RDW} \geq 15 \%$ & 7 & 7 & 14 & \\
\hline $\begin{array}{c}\text { Positive stool } \\
\mathrm{O} \text { and } \mathrm{P}\end{array}$ & 7 & 17 & 24 & \multirow{3}{*}{$p=0.176$} \\
\hline $\begin{array}{c}\text { Negative } \\
\text { stool } \mathrm{O} \text { and } \mathrm{P}\end{array}$ & 100 & 450 & 550 & \\
\hline \multicolumn{4}{|c|}{ *Statistically significant value at $p<0.05$} & \\
\hline
\end{tabular}




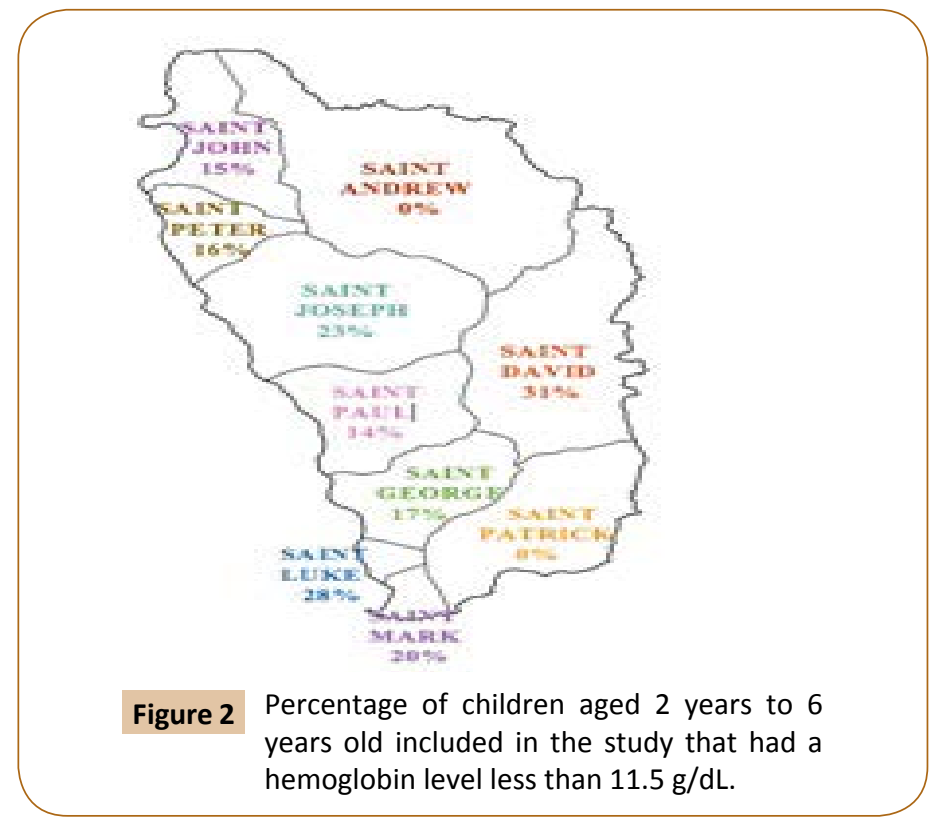

\section{Discussion}

In this study, we sought to determine the prevalence of anemia in preschool children aged two to six years old living in Dominica.

\section{Prevalence of anemia in Dominica}

Data from The World Bank determined that in 1990, 39.5\% of children under five years of age living in Dominica were anemic [7]. Over the following decade, the number of children diagnosed with anemia began to decline, with the lowest prevalence being $35 \%$ in the year 1999. Unfortunately, since 1999, the prevalence of childhood of anemia has been on the rise, with the latest value recorded as $36.5 \%$ in the year 2011 [8].

In this study, we deemed a hemoglobin value less than $11.5 \mathrm{~g} /$ $\mathrm{dL}$ as a determinant of anemia. Of the 595 children included in our study who had a hemoglobin value recorded, we found that $19.3 \%$ were considered to be anemic. According to the World Health Organization (WHO), a value of $19.3 \%$ is considered a mild level severity [9].

To help diagnose anemia in our sample population, we also recorded hematocrit, and red blood cell distribution width levels. The hematocrit level estimates the percentage of red blood cells in an individual's body. We deemed a hematocrit value less than $35 \%$ as a determinant of anemia. Our study found that $37.4 \%$ of children included in our study had a hematocrit level below $35 \%$.

The red blood cell distribution width estimates the variation in the red blood cell size and volume. This value is elevated when there is variation in red blood cell size, or volume, which is called anisocytosis. In our study, we deemed a value of $15 \%$ and greater, as a determinant of anemia. Our results concluded that $5.5 \%$ of children included in our study had a red blood cell distribution width greater than $15 \%$.

Although hemoglobin, hematocrit and red blood cell distribution width values are helpful in diagnosing anemia, there is no way to determine the exact type of anemia based on these values alone. The mean corpuscular volume (MCV) however, is very helpful in classifying anemia based on whether the value is low, normal, or high. Of the children who were considered to be anemic, $22.4 \%$ had a mean corpuscular volume less than $75 \mathrm{fL}$ indicating a microcytic anemia, $77.6 \%$ had a mean corpuscular volume between $76 \mathrm{fL}$ and $99 \mathrm{fL}$ indicating a normocytic anemia, and 0 children had a mean corpuscular volume greater than 100 $\mathrm{fL}$ indicating a macrocytic anemia.

Microcytic anemia is a broad category of anemia that encompasses the diseases of iron deficiency, alpha-thalassemia, beta-thalassemia, lead poisoning, sideroblastic anemia, anemia of chronic disease, and vitamin B6 deficiency. Normocytic anemia is another broad category that encompasses chronic diseases, as well as diseases of aplastic anemia, hemoglobinopathies such as sickle cell anemia, hereditary spherocytosis, glucose 6 phosphate dehydrogenase deficiency, pyruvate kinase deficiency, paroxysmal nocturnal hemoglobinuria, autoimmune conditions, microangiopathic anemia, and macroangiopathic anemia. Macrocytic anemia encompasses the deficiencies of vitamin B12 and folate.

Although there are numerous types of microcytic and normocytic anemia, the most common type of microcytic anemia found in children living in Dominica is probably iron deficiency anemia and alpha thalassemia. The most common type of normocytic anemia found in children living in Dominica is probably anemia of chronic infection and sickle cell disease.

Iron deficiency anemia is the most common type of anemia worldwide, and results from a decrease in iron. The decrease in iron could be caused by a plethora of factors but in children the most common cause is most likely due to malnutrition.

Alpha thalassemia occurs due to a defect in the alpha globin gene causing a decrease in alpha globin synthesis. This type of thalassemia is most common in Asian and African populations. There are four alpha globin genes and therefore three types of alpha thalassemia: [1]. Four gene deletion which occurs when no alpha globin is produced, and causes hydrops fetalis. This type of alpha thalassemia is incompatible with life [2]. Three gene deletion which results in the overproduction of beta globin produced, this type is called $\mathrm{HbH}$ disease and [3] one to two genes deleted which doesn't have a clinical significance.

Sickle cell anemia occurs when a point mutation occurs in an amino acid- the base glutamic acid is replaced with valine. When this occurs, the red blood cells begin to 'sickle,' which causes anemia and vaso-occlusive disease. This disease is also prevalent in African populations.

These various types of microcytic anemia are diagnosed based on additional laboratory markers. Those markers include serum iron, total iron binding capacity, ferritin, transferrin levels, \% transferrin saturation and hemoglobin $\mathrm{S}$. These markers are not routinely performed in children living in Dominica, and therefore, there is an additional fee to have this blood work performed. Without further testing it is extremely difficult to determine the exact variant of anemia these children suffer from. 


\section{Gender and age and anemia}

Of the 626 preschool children aged two to five years included in our study, $54.7 \%$ were female and $45.2 \%$ were male. We did not find any statistically significant differences in the prevalence of anemia based on gender.

Previous studies, also did not find a statistically significant difference in the prevalence of anemia based on gender. A three-phase descriptive cross-sectional study conducted in Cuba, recorded anemia prevalence rates in boys and girls at three different time periods-2005, 2008, and 2011. The last survey conducted in 2011, found an anemia prevalence of $26.5 \%$ in boys, and $25.5 \%$ in girls [2]. This was not statistically significant.

In our study, children were grouped into a category of less than 3.5 years old, and greater than 3.5 years old, to determine if there was a difference in the prevalence of anemia based on age. Although the majority of children included in our study sample, $60.6 \%$, were at least 3.5 years of age, we did not find any statistically significant differences in the prevalence of anemia based on age. Similarly, a cross sectional survey that was performed in Burma, on children aged 6 months to 36 months did not find a statistically significant difference in the prevalence of anemia based on age [4].

There are however some studies that depict age as being protective for the development of anemia. A cross sectional survey that was conducted in the Gaza Strip on children 2 years to 5 years old, found that hemoglobin concentration increased with age [3]. This was also found in a study conducted in Haiti, suggesting the difference was due to more varied diets in older children.

Another study performed in Haiti, a randomized controlled trial, examined male and female children between the ages of 3 years to 13 years. Although there was no statistically significant difference found in hemoglobin or anemia by sex, they did notice that age was protective for hemoglobin concentration and anemia [6]. The study also found that of the 1,167 children aged 3 years to 13 years that were enrolled in the study, preschool aged children had the lowest hemoglobin levels [6]. Additionally, another study that was conducted in Haiti showed $38.8 \%$ of children aged 6 months to 59 months were found to be anemic in association with young age, stunting, and low maternal hemoglobin status [6].

Various studies have found young children to be the most at risk population for the development of anemia. These conclusion stem from multiple factors probably due to: the presence of maternal anemia while the child is in utero, prolonged breastfeeding without iron supplementation, delayed onset of introduction of complementary foods, iron-poor diets, limited maternal education, and low socio-economic status. Many of these aforementioned factors exist in Dominica, and suggest the increased need for programs and policies to be implemented so that childhood anemia could be decreased.

\section{Preschool location and anemia}

Our study sample included 635 children-55\% female and $45 \%$ male, who attended preschool in all ten parishes of Dominica. We recorded the preschool attended by the children as well as the location, to determine if there were any differences in the prevalence of anemia based on parish.

Our study determined that the majority of the children attended preschool in the parish of St. George (58.7\%); however, the largest percentage of children who were anemic attended preschool in the parish of St. David (31\%). According to the 2011 Dominica census, the parish of St. George had a total population of 10,504, making it the largest populated parish in Dominica, whereas St. David had a total population of 3,248 individuals [10].

\section{Stool ova and parasites and anemia}

Infectious diseases such as those transmitted from hookworms, are very prominent in tropical environments like Dominica, where there is poverty, unsafe water, poor sanitation, and poor infrastructure. In our study, $4.4 \%$ of children had the presence of ova and/or parasites in their stool, which could lead to the development of iron deficiency anemia [11].

The two most common types of hookworm infections are Necator americanus and Ancylostoma duodenale. These worms make their way to an individual's gastrointestinal track and cause mechanical and chemical damage to the blood vessels. The hookworm then secretes anticlotting agents to thin the individual's blood, which provides nourishment for the worm [12]. Iron deficiency anemia results when the amount of blood ingested by the worm is greater than the amount of blood the individual is producing.

One way to combat iron deficiency anemia caused by hookworm infections could be providing deworming medication as well as iron supplementation. A meta-analysis of 14 randomized controlled trials conducting deworming practices in sub-Saharan Africa and Asia showed a significant increase in mean hemoglobin concentration, and an even greater response in individuals who were provided iron supplementation [1]. Iron supplementation can be an excellent source of added iron, especially when a caregiver is unaware a child is infected.

\section{Strengths and Limitations}

To our knowledge, this is one of the first descriptive studies to determine the prevalence of anemia among pre-school aged children living in Dominica. This study could be used as an initial study to generate more conclusive results in measuring the type of anemia that is affecting children under six years of age. One limitation to the study is that since we performed a non-concurrent medical chart review, and the health records of Dominican children (children of Dominica) are only available in paper version, variables we were interested in were not always available. Additionally, it is possible our sample was not representative of the entire pre-school population in Dominica. Another limitation of the study is that we were unable to determine the specific type of anemia. 


\section{Conclusion}

The data in this study demonstrate that childhood anemia in Dominica is a public health issue that needs to be addressed before it is too late in a child's growth and development. Future studies, aimed at determining the type of anemia affecting children under six years old living in Dominica, should be conducted. These studies should include measuring additional serum markers such as serum iron, total iron binding capacity, ferritin, transferrin levels, \% transferrin saturation, and hemoglobin $S$ so that the exact type of anemia could be diagnosed, and strategies could

\section{References}

1 Balarajan Y, Ramakrishnan U, Özaltin E, Shankar, AH, Subramanian SV (2011) Anaemia in low-income and middle-income countries. The Lancet 378: 2123-2135.

2 Rodríguez PGM, Jiménez S, Basabe B, García RG, Macías C, et al. (2014) Anemia in children under five years old in Eastern Cuba, 2005-2011. Medicc Review 16: 16-23.

3 El Kishawi RR, Soo KL, Abed YA, Muda W (2015) Anemia among children aged 2-5 years in the gaza strip-palestinian: A cross sectional study. BMC Public Health, 15.

4 Zhao A, Gao H, Li B, Zhang J, Win NN, et al. (2016) Inappropriate feeding behavior: One of the important causes of malnutrition in 6- to 36-month-old children in Myanmar. Am J Trop Med Hyg 95: 702-708.

5 McLean E, Cogswell M, Egli I, Wojdyla D, De Benoist B (2009) Worldwide prevalence of anaemia, WHO vitamin and mineral be developed to decrease this childhood burden. Strategies that could be implemented to decrease the prevalence of anemia are: 1) providing nutrient, specifically iron, rich food in the home, as well as the school; 2) timely introduction of iron-rich foods; 3) nutrient-fortified infant formula and iron enriched infant cereal as a supplement to breast milk; and lastly 4) ensure pregnant women are receiving proper nutrition as well as iron supplementation for the duration of their pregnancy. Diagnosing anemia as early as possible is of utmost importance since iron is a critical nutrient for neonatal growth and development.

nutrition information system, 1993-2005. Public Health Nutr 12 444-454.

6 Iannotti LL, Delnatus JR, Odom AR, Eaton JC, Griggs JJ, et al. (2015) Determinants of anemia and hemoglobin concentration in Haitian school-aged children. Am J Trop Med Hyg 93: 1092-1098.

7 Zhao A, Zhang Y, Peng Y, Li J, Yang T, et al. (2012) Prevalence of anemia and its risk factors among children 6-36 months old in Burma. Am J Trop Med Hyg 87: 306-311.

8 http://data.worldband.org/indicator/SHA.ANM.CHLD.ZS?page=1

9 http://apps.who.int/iris/bitstream/10665/43894/1/9789241596657_ eng.pdf

10 www.dominica.gov.dm/cms/files/2011_census_report.pdf

11 https://data.unicef.org/wp-content/uploads/country_profiles/ Dominica/Maternal_DMA.pdf

12 Centre for Disease Control and Prevention (2014) Global Health - Division of Parasitic Diseases, GA, USA. 